\title{
Research on the Path of Typical Region to Undertake Industrial Transfer in Beijing-Tianjin-Hebei- — Taking the Inner Mongolia-Shanxi-Hebei Great Wall Golden Triangle Cooperation Zone as an example
}

\author{
Wang Ting \\ Business School, Shanxi Datong University, Datong, Shanxi, China
}

Keywords: Beijing-Tianjin-Hebei Industrial Transfer; path analysis; the Inner Mongolia -Shanxi-Hebei Great Wall Golden Triangle Cooperation Zone

\begin{abstract}
In the context of the coordinated development of Beijing, Tianjin and Hebei, the Inner Mongolia-Shanxi-Hebei Great Wall Golden Triangle Cooperation Zone is taken as an example to study the regional industrial undertaking capacity and industrial transfer path. On the basis of the full analysis of the development status and the situation of industrial transfer and development of the Inner Mongolia-Shanxi-Hebei Great Wall Golden Triangle Cooperation Zone, this paper analyzes the path research on the industrial transfer of Beijing, Tianjin and Hebei Province in the typical areas, excavates problems in the actual process and solves them gradually, and gives corresponding policy suggestions.
\end{abstract}

\section{The Development Advantages of the Inner Mongolia-Shanxi-Hebei Great Wall Golden Triangle Cooperation Zone}

The strategy of the Neimenggu-Shanxi-Hebei Great Wall Golden Triangle Cooperation Zone is a joint strategy proposed by Wulanchabu, Datong and Zhangjiakou (hereinafter referred to as Wu-Da-Zhang) governments to borrow the integrated power of Beijing, Tianjin and Hebei and strive for the strategy of differentiation policy ${ }^{[1]}$. At present, with the joint promotion of the three parties, this regional strategy has been formally included in the national "The Belt and Road" opening-up strategic plan. At present, he Neimenggu-Shanxi-Hebei Great Wall Golden Triangle Cooperation Zone faces a good development opportunity. The regional strategy promotes the Neimenggu-Shanxi-Hebei Great Wall Golden Triangle Cooperation Zone to develop into a important economic zone after Shijiazhuang and Tangshan in addition to Beijing and Tianjin. First of all, there are advantages of resource integration in the three cities. The Neimenggu-Shanxi-Hebei Great Wall Golden Triangle Cooperation Zone is located in a good regional cooperation circle and has rich cultural and economic connotations. It plays an important role in serving and safeguarding the development of Beijing-Tianjin-Hebei. The cooperation between Wulanchabu City, Datong City and Zhangjiakou City is a $1+1+1>3$ model. The development of cultural industry, population superiority and environmental problems are integrated and developed, the integration of economic and social benefits has been realized, and the comprehensive competitiveness and overall strength of the region have been greatly improved ${ }^{[2]}$.

Second, the support of national policies. In 2015, General Secretary Xi Jinping officially included the Neimenggu-Shanxi-Hebei Great Wall Golden Triangle Cooperation Zone into the Beijing-Tianjin-Hebei coordinated development plan at the Political Bureau of the Central Committee of the CPC meeting.This means that the development of the Neimenggu-Shanxi-Hebei Great Wall Golden Triangle Cooperation Zone has risen to an important position in the national strategic development. Later, the formulation of relevant policies and the development strategies designated by the three cities according to the field survey from the perspective of policies and actual conditions promoted the development of the Neimenggu-Shanxi-Hebei Great Wall Golden Triangle Cooperation Zone and the optimization of industrial structure. This drives the rapid development of the private economy. This major development model is a model of "party and government promotion, market-driven, and civil-linked", and continues to advance with the support 
of the government, the market, and the people.

Finally, there is a clear orientation of development. The development of the Neimenggu-Shani-Hebei Great Wall Golden Triangle Cooperation Zone is the development after the field investigation. It fully grasps the development status of the Neimenggu-Shani-Hebei Great Wall Golden Triangle Cooperation Zone, grasps the weak points and advantages of development, and effectively integrates the resources of the three cities. At the same time, it also promotes industrial communication and complementary development between the three cities, and promotes the complementary advantages between resources. This will help to break the marginal predicament of the Niemenggu-Shanxi-Hebei Great Wall Golden Triangle Cooperation Zone, and also develop the resource advantages of the three cities, and vigorously develop industries with regional characteristics, such as characteristic agriculture and animal husbandry, cultural tourism, etc. In addition, it will help to achieve centralized poverty alleviation work, integration of industrial resources, and promote closer economic ties between the three cities. In this way, the poverty alleviation work of the three cities can be linked together to carry out all-round poverty alleviation work and reduce the cost of poverty alleviation, and this can also drive poverty alleviation work in the whole region and find a new development path ${ }^{[3]}$.

\section{Analysis of Industrial Transfer Ability of Inner Mongolia-Shanxi-Hebei Great Wall Golden Triangle Cooperation Zone}

\subsection{Cross-regional financial cooperation}

Cross-regional financial cooperation is the driving force for regional development and an important force to promote rapid economic development ${ }^{[4]}$. The Neimenggu-Shanxi-Hebei Great Wall Golden Triangle Cooperation Zone is a process of constructing regional economic integration. In the process of regional construction, we should focus on the development of regional economic construction, do a good job in industrial transformation, develop some key industries in the region, draw closer to each other, make up the weak and inadequate areas in many economic areas, promote the development and construction of new industries, and promote the new urbanization and modern ecological nomadism and the development of some cultural tourism industries, promote the construction of ecological environment, promote the unification of economic and social benefits, promote the harmonious and rapid development of the economy, and promote the optimal development of the industrial economy. In addition, in terms of resource integration, the alliance between regions can break the geographical restrictions of finance, improve the services and innovation of other financial industries, improve the flexibility of financial services, promote the cross-regional development of financial services, and promote finance in a better direction.

\subsection{Financial development potential}

The Neimenggu-Shnxi-Hebei Great Wall Golden Triangle Cooperation Zone has a certain industrial advantage and financial development potential to undertake the industrial transfer of Beijing-Tianjin-Hebei. This is mainly reflected in the industrial upgrading and optimization cooperation in the cooperation zone. From the perspective of overall planning, the Neimenggu-Shanxi-Hebei Great Wall Golden Triangle Cooperation Zone can undertake the relevant technologies of the integrated development industry of Beijing-Tianjin-Hebei, and can also promote the development of the cooperation zone towards eco-environmental protection, and establish a financial, green and innovative development area. This is the great potential for the development of the Neimenggu-Shanxi-Hebei Great Wall Golden Triangle Cooperation Zone. It is an important force for promoting regional financial development. It can develop on this basis, promote the establishment and development of regional economy, and promote the development and innovation of science and technology, and further improve the environment for financial development to promote the undertaking of the Neimenggu-Shanxi-Hebei Great Wall Golden Triangle Cooperation Zone and the Beijing-Tianjin-Hebei region, continuously open up new development paths, promote regional finance to develop toward a larger direction, stimulate new development vitality, and 
promote open development of regional financial economy.

\subsection{Optimal allocation of resource elements}

The Neimenggu-Shanxi-Hebei Great Wall Golden Triangle Cooperation Zone undertakes the industrial transfer of Beijing-Tianjin-Hebei, which can stimulate new vitality for industrial development, promote industrial transformation in the new normal, and promote more stable and healthy economic development ${ }^{[5]}$. This is a great development opportunity. The joint region will promote the effective integration and flow of economic factors in the three places, promote the effective allocation of resource factors, realize regional resource integration, and promote market integration and development. Establishing the advantages of local resources development, building up industries with regional characteristics and to enhance market mobility. Whether it is the agriculture and animal husbandry culture of Wulanchabu, the resource advantage of Datong, Shanxi, or the natural customs of Zhangjiakou, the comprehensive arrangement of these regional economic factors promotes the optimal allocation of economic development. Resource factors are an important basis for promoting regional development, while regional cooperation can promote the optimal integration of regional resources. Therefore, we must actively promote inter-regional financial cooperation, open up a variety of economic development roads, improve resource utilization efficiency, and optimize resource allocation.

\section{The Choice of Industrial Transfer Path in Inner Mongolia-Shanxi-Hebei Great Wall Golden Triangle Cooperation Zone}

\subsection{Financial resources}

It takes certain financial resources to undertake the industrial transfer of Beijing-Tianjin-Hebei. Those industries will consider the transfer of industries according to the geographical location or resource characteristics of the region, such as some ports, labor-intensive industries, resource-based industries, etc. These are all considered in the context of industrial transfer. The economy is an important foundation for regional development and an important factor in determining whether a region can be developed. The Neimenggu-Shanxi-Hebei Great Wall Golden Triangle Cooperation Zone has a very obvious advantage that is supported by national policies. The government's strong support can remove most of the obstacles for the development of the industry. Moreover, the Neimenggu-Shanxi-Hebei Great Wall Golden Triangle Cooperation Zone has policy support and protection. Policy support is an important support for promoting the rapid development of the economy in industrial transfer development zones. Policy support promotes the improvement and development of the market. And the government can make maximum guidance in the cooperation zone to promote resources in a more equitable direction.

\subsection{Industrial innovation}

Industrial transfer is not only a high demand for industrial transfer areas, it also has certain requirements for the future development of the industry. With the impetus of the policy, the Neimwnggu-Shanxi-Hebei Great Wall Golden Triangle Cooperation Zone can innovate and promote the upgrading and transformation of related industries and promote the improvement of industrial capabilities. Industrial transfer is not the purpose of the industry's continued development. The fundamental of industrial development is continuous innovation and promoting sustainable development of the industry. Industrial innovation is not a purely innovative work, but an innovation and development based on the integration and development of industries and transfer areas. Although this process is difficult and uncontrollable, the regional industrial transfer must adhere to industrial innovation, so that the industrial transfer can be successfully completed. In addition, when industrial innovation is in place, we must adhere to the correct path of innovation, that is, to carry out innovation work according to the characteristics and actual conditions of industrial development. For example, the innovative ways of labor-intensive industries and resource-intensive industries are completely different. It is necessary to start the industrial 
upgrading and innovation work from the actual situation, promote the systematic development of the industry, and finally establish an industrial development chain to promote the rapid development and improvement of the economy.

\subsection{City positioning}

Do a good job in positioning the city. The city of Neimenggu-Shanxi-Hebei Great Wall Golden Triangle Cooperation Zone has distinctive development characteristics. The farming and pastoral culture of Wulanchabu, the resource advantages of Datong in Shanxi, the natural customs of Zhangjiakou, etc. These are the characteristics of the city and the best way to develop the city. A city that wants to really develop must have a clear positioning, and know how to develop ${ }^{[6]}$. For example, Wulanchabu can start from the characteristics of Neimenggu. Agriculture and animal husbandry is its characteristic industry. When striving to develop characteristic industries, it can also promote the development of other industries, such as the development of tourism. Speaking of the development of tourism, the best in tourism in these three cities is Zhangjiakou City. At this time, we can give full play to the resource advantages of centralized and integrated resources, and promote development in a comprehensive way. Zhangjiakou City can promote the development of ecotourism in Wulanchabu City. Datong City is the most resource-rich city among the three cities, and can effectively develop a resource-intensive economy and establish a resource center. The positioning of cities is the main foundation for urban establishment and development ${ }^{[7]}$.

After the completion of urban positioning, it is possible to undertake the industrial transfer work of Beijing-Tianjin-Hebei according to the different characteristics of the city, and form a city with comprehensive development of modern strategic industry and modern service industry. Taking full advantage of the characteristics of the city for development, whether it is tourism, nomadic or pension industry, these can fully develop the green industry. Datong can use the advantages of distance and the resource advantages of its own development to seek the development of industry, and carry out the transfer work with some industries of Beijing-Tianjin-Hebei, and vigorously develop the industry; Zhangjiakou City can use the urban civilization and the city's tourism service industry to link up with the Beijing-Tianjin-Hebei service industry to promote industrial innovation and development, and promote the progress of urban construction; Wulanchabu City can be developed as a city to promote ecological civilization construction. Due to the rapid economic development, the Beijing-Tianjin-Hebei region has paid a great price in the environment. Therefore, some eco-tourism and environmental protection industries can be connected to Wulanchabu City to display a distinctive and folk culture, to create a tourism brand and promote the cooperation and development of the cultural tourism industry.

\section{Acknowledgements}

Project Name: Soft science research project of Shanxi Province "Research on the industry linkage development stratagem of the Great Wall Golden Triangle Cooperation Zone.” Project Number: 2016041016-4.

\section{References}

[1] Research group of Wulanchabu Central Sub- Branch of People's Bank of China. Study on the Strategic Development of Regional Economic Integration of the Neimenggu-Shanxi-Hebei Great Wall Golden Triangle Cooperation Zone [J]. Northern Finance Journal, 2015, (9):33-36.

[2] Research group of Baoding Central Sub- Branch of People's Bank of China. Research on the Capacity to Undertake Industrial Transfer in the Context of the Coordinated Development of Beijing Tianjin Hebei -- Taking Baoding City of Hebei Province as an Example [J].Hebei Finance. 2017, (9):38-43.DOI:10.3969/j.issn.1006-6373.2017.09.010.

[3] Fenglian Zhang. Research on Hebei province undertaking industrial transfer in the background of coordinated development of Beijing Tianjin Hebei [J].Bussiness, 2016, (1):253. 
[4] Raygang Sun. Group Integration into Beijing Tianjin Hebei Mutual Complement and Common Development -- Thinking about Building the Neimenggu-Shanxi-Hebei (Wu-Da-Zhang) Great Wall Golden Triangle Economic Cooperation Zone [J]. Journal of Practice (theory edition), 2016, (5):32-33.

[5] Yongling Yao, Ruoyu Li. Regional Economic Effects of Beijing-Tianjin-Hebei Industrial Transfer [J]. Economy and Management, 2017, 31(6):17-23.

[6] Jianfeng Wang, Yan Lu. An Empirical Study on the Comprehensive Effect of Regional Industrial Transfer in Beijing, Tianjin and Hebei [J]. Journal of Hebei University of Economics and Trade, 2013, 34(1):81-84.

[7] Liping Tian, Hao Liu, Jian Gao, etc. Evaluation and Path Selection of Undertaking Industry Transfer Capability in Hebei Province_-Based on the Perspective of Beijing-Tianjin-Hebei Cooperative Development [J]. Journal of Commercial Economics, 2016, (20):201-204. 